\title{
Picking apart polyubiquitin chains
}

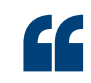

Polyubiquitin-

linkage-specific antibodies...

now make

it possible

to easily

discriminate

between

the different

chains.
Polyubiquitin chains are attached to proteins to mediate various cellular processes - Lys63-linked chains often have signalling functions, whereas Lys48-linked chains are associated with protein degradation. Practical limitations, however, have hampered our ability to tease apart the subtler differences between Lys63- and Lys48-linked chains. Polyubiquitin-linkage-specific antibodies, developed by Newton and colleagues, now make it possible to easily discriminate between the different chains. Studies with these antibodies suggest that polyubiquitin editing might be a general mechanism for attenuating signalling.

Previously, researchers relied on mass spectrometry or mutational studies to study the differences between Lys63- and Lys48-linked chains. These approaches, however, have considerable limitations: mass

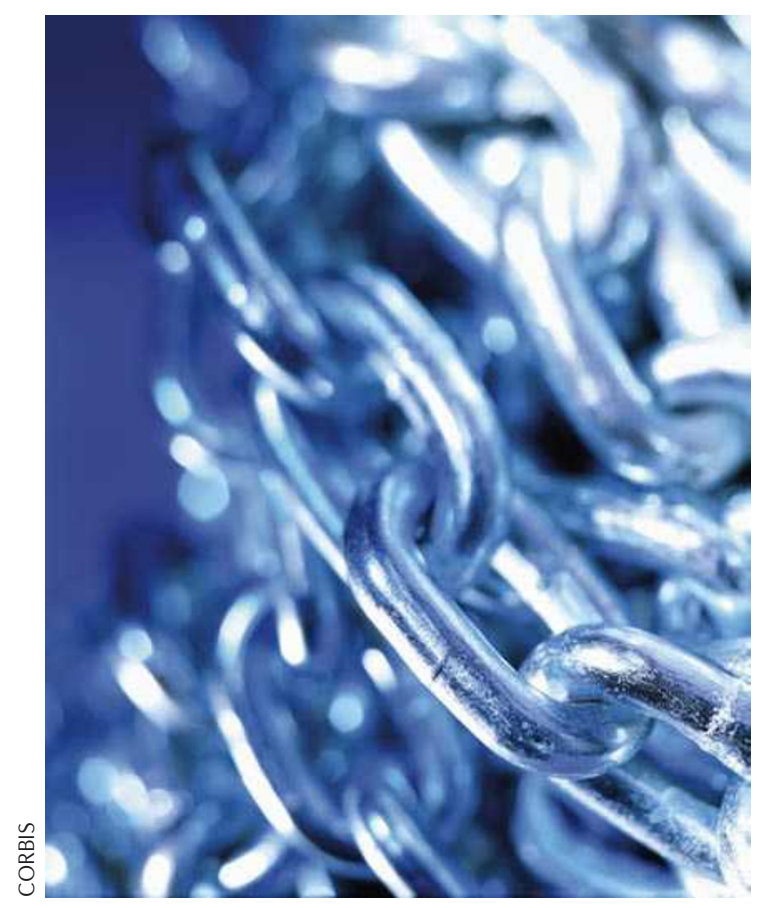

spectrometry requires expensive equipment, whereas mutant ubiquitin might not accurately recapitulate in vivo modifications, and neither approach is practical for examining the rapid changes in ubiquitylation over time. Thus, Newton and colleagues developed Lys48- and Lys63-linkage-specific antibodies, and showed that these antibodies are highly specific and can be used for immunoprecipitation and western blotting.

These antibodies can provide valuable insights into polyubiquitinchain biology. For instance, using indirect immunofluorescence microscopy, the authors demonstrated that Lys48-linked chains localize to the nucleus and the cytoplasm of HeLa cells, but are less abundant in the nucleoli of the nucleus, which coincides with proteasomal labelling. By contrast, Lys63linked chains localize at cytoplasmic speckles, which does not correspond to proteasomal labelling. These findings support the notion that Lys48 linkages, but not Lys63 linkages, are crucial for protein degradation.

To further demonstrate the usefulness of these antibodies, the authors examined the polyubiquitylation of the kinase adaptor RIP1, which is essential for nuclear factor- $\kappa \mathrm{B}$ $(\mathrm{NF}-\kappa \mathrm{B})$ activation. Following treatment with tumour-necrosis factor (TNF), RIP1 is recruited to TNF receptor-1 (TNFR1). Lys63-linked polyubiquitylation of RIP1 has been suggested to mediate downstream signalling and activation of NF- $\mathrm{KB}$. Using their antibodies, Newton and colleagues demonstrated that 5 minutes after TNF treatment, RIP1 is modified almost exclusively with Lys63-linked chains. 10 minutes after TNF treatment, however, RIP1 has fewer Lys63-linked chains and many more Lys48-linked chains. As Lys48-linked chains target proteins for degradation, polyubiquitylation editing could therefore induce the degradation of activated RIP1, thereby attenuating signalling. Similarly, the authors demonstrate that IRAK1, which participates in innate immunity signalling, is initially polyubiquitylated with Lys63-linked chains, but later becomes polyubiquitylated with Lys48-linked chains.

Taken together, these findings indicate that polyubiquitin-chain editing might represent a general mechanism of attenuating signalling. The authors further suggest that "these reagents hold the same potential as antibodies specific for phosphorylated amino acids in revealing the rapid changes in posttranslational modifications that accompany signaling."

Asher Mullard

ORIGINAL RESEARCH PAPER Newton, K. et al. Ubiquitin chain editing revealed by polyubiquitin linkage-specific antibodies. Cell 134, 668-678 (2008) 